\title{
The 1st INTEGRAL SPI-ACS gamma-ray burst catalogue ${ }^{\star}$
}

\author{
A. Rau ${ }^{1}$, A. v. Kienlin ${ }^{1}$, K. Hurley ${ }^{2}$, and G. G. Lichti $^{1}$ \\ 1 Max-Planck-Institut für extraterrestrische Physik, Postfach 1312, 85741 Garching, Germany \\ e-mail: arau@mpe.mpg.de \\ 2 UC Berkeley Space Sciences Laboratory, Berkeley, CA 94720-7450, USA
}

Received 30 March 2005 / Accepted 15 April 2005

\begin{abstract}
We present the sample of gamma-ray bursts detected with the anti-coincidence shield ACS of the spectrometer SPI on-board INTEGRAL for the first 26.5 months of mission operation (up to Jan. 2005). SPI-ACS works as a nearly omnidirectional gamma-ray burst detector above $\sim 80 \mathrm{keV}$ but lacks spatial and spectral information. In this catalogue, the properties derived from the $50 \mathrm{~ms}$ light curves (e.g., $T_{90}, C_{\max }, C_{\mathrm{int}}$, variability, $V / V_{\max }$ ) are given for each candidate burst in the sample. A strong excess of very short events with durations $<0.25 \mathrm{~s}$ is found. This population is shown to be significantly different from the short- and long-duration burst sample by means of the intensity distribution and $V / V_{\max }$ test and is certainly connected with cosmic ray hits in the detector. A rate of 0.3 true gamma-ray bursts per day is observed.
\end{abstract}

Key words. gamma rays: bursts - catalogs - instrumentation: detectors

\section{Introduction}

Although discovered more than three decades ago (Klebesadel et al. 1973), the phenomenon of cosmic gamma-ray bursts (GRB) is still challenging, with many open questions to solve. Therefore, the detection and investigation of GRBs is one of the important scientific objectives of ESA's International GammaRay Astrophysics Laboratory (INTEGRAL) mission (Winkler et al. 2003). INTEGRAL contributes to GRB science in two ways. (i) For bursts which occur in the field of view (FoV) of the spectrometer SPI (Vedrenne et al. 2003) and of the imager IBIS (Ubertini et al. 2003), INTEGRAL provides accurate positions ( 2 arcmin) for rapid ground- and space-based follow-up observations. In addition, high-energy spectra in the $20 \mathrm{keV}-8 \mathrm{MeV}$ range are recorded (see Mereghetti et al. 2004 for a recent summary). (ii) The anti-coincidence system of SPI (SPI-ACS) acts as a nearly omnidirectional GRB detector in the $\sim 80 \mathrm{keV}$ to $\sim 10 \mathrm{MeV}$ energy range (von Kienlin et al. 2003).

It has long been known that there are two distinct classes of GRBs which differ observationally in duration and spectral properties (Mazets et al. 1981; Norris et al. 1984; Dezalay et al. 1992; Hurley 1992; Kouveliotou et al. 1993; Norris et al. 2000). This was quantified using data from the Burst and Transient Source Experiment (BATSE) detectors (Fishman et al. 1989) on-board NASA's Compton Gamma-ray Observatory (CGRO). The sample of more than one thousand bursts included in the 4th BATSE catalogue displayed a clear bimodal duration

* Table 2 is only available in electronic form at the CDS via anonymous ftp to cdsarc.u-strasbg.fr (130.79.128.5) or via http://cdsweb.u-strasbg.fr/cgi-bin/qcat?J/A+A/438/1175 distribution, with one group having short durations $(<2 s)$ and the other longer durations (seconds to minutes) (Kouveliotou et al. 1993). Statistically, the peak energy of the Band spectrum for short bursts (Band et al. 1993), $E_{\mathrm{p}}$, is higher (typically $\sim 100-1000 \mathrm{keV}$ ) than that of the long-duration events (typically $\sim 70-500 \mathrm{keV}$ ) (Fishman \& Meegan 1995). The sensitivity of the anti-coincidence system of SPI to the short/ hard burst population is nearly unprecedented, which raised expectations before the launch of the mission that new insights into the distribution of short bursts would be achieved.

In this paper we present the 1st catalogue of gamma-ray bursts detected by SPI-ACS. We describe the instrumentation and data (Sect. 2), the sample selection criterion (Sect. 3) and the sample analysis (Sect. 4). In Sect. 5 the duration- and intensity distributions of the sample are discussed.

\section{Instrumentation and data}

The anti-coincidence system ${ }^{1}$ of SPI consists of 91 Bismuth Germanate (BGO) crystals with a total mass of $512 \mathrm{~kg}$. The thickness of the individual crystals ranges from 16 to $50 \mathrm{~mm}$. They are positioned in two rings (the upper and lower collimator ring) whose axes are along the viewing direction of the spectrometer, between the coded mask and the detector plane of SPI; in addition, there are side-shield and rear-shield assemblies (Fig. 1). Incoming $\gamma$-rays are converted into optical photons with a wavelength of $\sim 480 \mathrm{~nm}$. Each BGO is redundantly monitored by two photomultiplier tubes. In addition to

1 Developed at the Max-Planck Institute for extraterrestrial Physics, Garching, Germany. 


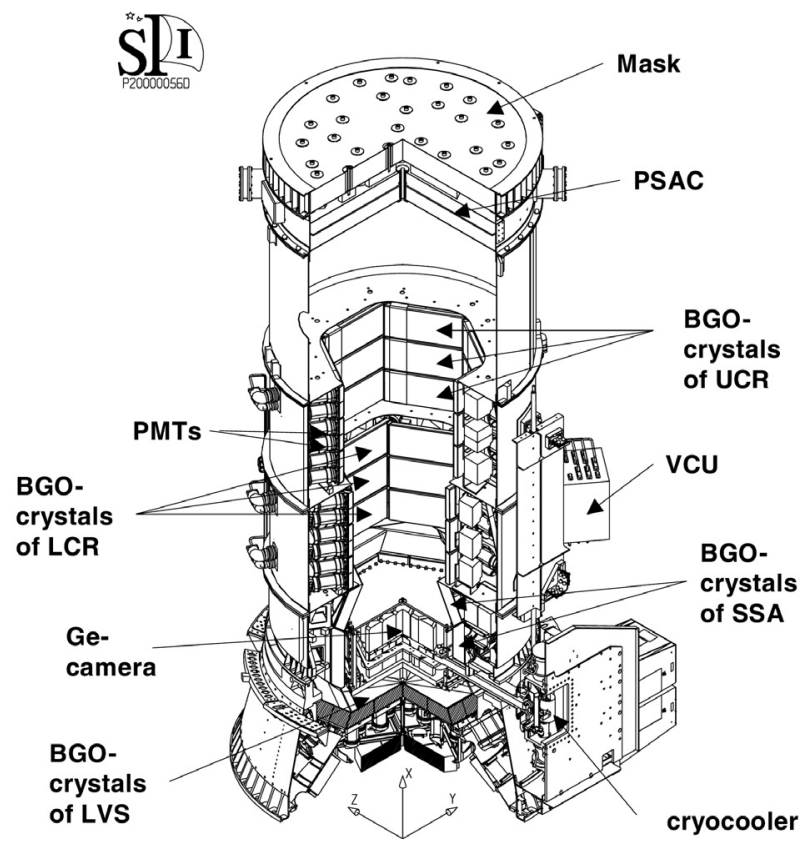

Fig. 1. Spectrometer SPI and SPI-ACS. The SPI-ACS consists of the plastic scintillator (PSAC) and the BGO crystals of the upper (UCR) and lower collimator rings (LCR) plus the side-shield assembly (SSA) and lower veto shield (LVS). The crystals are monitored by the photomultiplier tubes (PMT) and the events are processed in the veto control unit (VCU).

the BGO, a plastic scintillator (PSAC), whose purpose is the reduction of the $511 \mathrm{keV}$ background produced by particle interactions in the passive mask, is located directly below the coded mask.

Since the ACS encloses the SPI, it provides a quasiomnidirectional field of view with a large $\left(\sim 0.3 \mathrm{~m}^{2}\right)$ effective area for the detection of gamma-rays (von Kienlin et al. 2003a). Unfortunately, the electronics does not allow for good spatial resolution; the burst data consist only of the total event rate from all the crystals (for more details see Lichti et al. 2000) with a time resolution of $50 \mathrm{~ms}$. In addition, however, each BGO crystal is read out every $96 \mathrm{~s}$ with an integration time of $1 \mathrm{~s}$, which in principle allows a rough position estimate for long and bright events.

The SPI-ACS data do not include spectral information because the energy of the gamma-rays is not measured. The only information which is avaiable is that the energy of the interacting photon must be above the threshold energy of $\sim 80 \mathrm{keV}$. Due to the different properties of the individual crystals, their associated photomultipliers, and the redundancy concept (signals from two different crystals are fed to the same front-end electronics) this energy threshold can only be estimated very coarsely.

As a veto shield the SPI-ACS has no upper limit to the energy range (von Kienlin et al. 2003b). Therefore, the physical properties of individual events (e.g., peak flux, fluence) cannot be estimated directly from the data. A conversion from detector counts to photons $\mathrm{cm}^{-2} \mathrm{~s}^{-1}$ is possible only for bursts whose arrival directions and spectra (e.g., the Band function parameters - Band et al. 1993) are accurately known from other gamma-ray instruments. For these bursts the effective area of the detector can be modelled by simulations of the photon transport in the instrument as a function of the incident angle and energy. While the number of GRBs for which this method can be applied is very small, it was especially useful for the determination of the SPI-ACS spectral parameters for the giant flare from SGR 1806-20 on Dec. 272004 (Mereghetti et al. 2005).

SPI-ACS is part of the INTEGRAL Burst Alert System (IBAS; Mereghetti et al. 2004). A software trigger algorithm searches for an excess in the overall count rate with eight different time binnings $(0.05,0.1,0.2,0.4,0.8,1,2$, and $5 \mathrm{~s})$ with respect to a running average background. The trigger thresholds depend on the time binning and are set to 9, 6, 9, 6, 9, 6, 9 and $6 \sigma$, respectively. For each trigger an ASCII light curve ( $5 \mathrm{~s}$ pre-trigger to $100 \mathrm{~s}$ post-trigger) and the spacecraft ephemeris are stored and made publicly available ${ }^{2}$. It was originally planned to distribute transient detections automatically. However, due to the large number of spurious triggers produced by solar flares, cosmic rays and radiation belt passages, SPI-ACS alerts are now sent only manually to the interested community. During the early mission phase many spurious triggers were produced by rate increases in single $50 \mathrm{~ms}$ time bins ( $\sim 15$ trigger per hour). A software solution was found to remove these events from the data stream.

Since December 2002 SPI-ACS has been an important member of the 3rd Interplanetary Network (IPN) of gammaray burst detectors, which provides burst localizations using the triangulation method (Hurley 1997). Thus the lack of spatial resolution of the ACS can at least partly be compensated for. By studying triangulations of a number of bursts with precisely known localizations together with Konus/Wind and/or Helicon/Coronas- $F$ instruments the absolute timing of the instrument was adjusted and verified to an uncertainty of $25 \mathrm{~ms}$ (Rau et al. 2004).

\section{Sample selection}

A sophisticated statistical analysis of the GRB events in the SPI-ACS overall count rate requires a thorough and robust sample selection. Generally, the best method to distinguish gammaray bursts from other events occurring in the data stream is their localization in celestial coordinates. Likewise, detections of the events by other missions with gamma-ray detectors, e.g. the instruments participating in the IPN, can be used to discriminate between real cosmic events and those with a solar or particle origin. Due to the lack of spatial information in the SPI-ACS data, observations with independent instruments are required in order to apply this localization-based selection criterion.

Due to the different energy ranges, sensitivities, and parts of the sky observed by the various currently active gamma-ray detectors, the above-mentioned method of selection applies only to a sub-sample of the GRBs observed with SPI-ACS. Due to its nearly omnidirectional field of view and sensitivity, a considerable fraction of GRBs is expected to be detected only by SPI-ACS. Thus, in order to study a statistically more significant

${ }^{2}$ http://isdcarc.unige.ch/arc/FTP/ibas/spiacs/ 
sample, we defined our selection criteria to be independent of the confirmation by other instruments and based only on the SPI-ACS light curve.

The base sample was constructed from all triggered events in the SPI-ACS overall rate light curve. Each trigger in this preliminary sample was subsequently checked for particle or solar origin using the INTEGRAL Radiation Monitor (IREM) and the Geostationary Operational Environmental Satellites $(\mathrm{GOES})^{3}$, respectively. Triangulated events with a localization consistent with the position of a Soft Gamma Repeater (SGR) were removed from the sample. A significance threshold was then applied to each event with a possible cosmic origin, such that a trigger was included in the sample of GRB candidates when its significance $S$ above the background $B$ exceeded $S=12 \sigma$ in any time interval during the event. Here, $1 \sigma$ corresponds to $1.57 \times \sqrt{B}$, where the factor 1.57 takes into account the measured deviation of the background from a Poissonian distribution (von Kienlin et al. 2003b, Ryde et al. 2003). The conservative threshold of $S=12 \sigma$ has been chosen in order to minimize the contamination by weak events of non-GRB origin. Examples of candidate GRBs from the final sample are shown in Fig. 2.

Based on the selection criteria described above, a total of 374 candidate GRBs were detected between Oct. 27, 2002 and Jan. 12, 2005. In addition, 14 GRBs with a significance below the selection threshold, but confirmed by other gamma-ray missions, were detected. From the total sample of 388, a cosmic origin for 179 has been confirmed. Most of these events were detected by Konus/Wind and/or Ulysses. Unfortunately, the Ulysses GRB experiment had to be turned off in December 2003 due to the diminishing output of the radioisotope thermoelectric generator.

A summary of the currently active missions and the confirming detections of SPI-ACS candidate GRBs is presented in Table 1. The number of events detected by SPI-ACS and HETE-2 is relatively low compared to the total number of GRBs detected by HETE-2 during the INTEGRAL mission time. This is not surprising; it is the result of the low sensitivity of SPI-ACS to X-ray flashes and X-ray rich bursts, which constitute approximately $2 / 3$ of the HETE-2 GRB sample (Lamb et al. 2005). These events are characterized by an $\mathrm{E}_{p}<80 \mathrm{keV}$ and thus have their emission maximum below the SPI-ACS energy range. The number of bursts detected both in the INTEGRAL FoV and SPI-ACS is very low as well, since the effective area of SPI-ACS is a minimum for events irradiating the satellite from that direction.

Taking into account the observation time losses due to radiation belt passages and regular SPI detector annealings, SPIACS was actively observing GRBs for a total 21.6 months up to Jan. $12,2005^{4}$. This time corresponds to a rate of 216 candidates and 92 confirmed bursts per year selected according to

\footnotetext{
${ }^{3}$ http://www. sec.noaa.gov

4 The INTEGRAL satellite is in a highly elliptical orbit with a period of $72 \mathrm{~h}$. Due to the high particle flux the instruments are deactivated during the perigee passage and are online only for $\sim 62 \mathrm{~h}$ per orbit. Approximately twice a year, the SPI Germanium detectors need to be heated up and cooled again (annealing) to counter the
}

Table 1. Gamma-ray burst detectors and satellites and number of reported simultaneous burst detections with SPI-ACS. (1): single photon counting, (2): $64 \mathrm{~ms}$ resolution during flare mode.

\begin{tabular}{lccc}
\hline \hline Instrument & $\begin{array}{c}\text { Energy } \\
{[\mathrm{keV}]}\end{array}$ & $\begin{array}{c}\text { Time res. } \\
{[\mathrm{s}]}\end{array}$ & Conf. \\
\hline Konus/Wind & $10-10000$ & $0.002-2.39$ & 164 \\
Ulysses & $25-150$ & $0.008-2.0$ & 92 \\
Mars Odyssey & $30-8000$ & $0.032-0.25$ & 79 \\
HETE-2 & $6-400$ & $(1)$ & 42 \\
RHESSI & $3-20000$ & $(1)$ & 79 \\
INTEGRAL FoV & $3-15000$ & $(1)$ & 4 \\
Helicon/Coronas-F & $10-10000$ & $(2)$ & 3 \\
Swift & $15-350$ & $(1)$ & 3 \\
RXTE/ASM & $1.5-12$ & 100 & 2 \\
\hline
\end{tabular}

our criteria. For comparison, Lichti et al. (2000) predicted the rate of GRBs prior to the start of INTEGRAL to be $\sim 160$ per year at a $10 \sigma$ level.

\section{Sample analysis}

For each candidate GRB in the sample, a linear fit to the background in a time interval before and after the event was performed and the resulting background counts subtracted. Next, a correction for dead time in the detector and electronics $(<3 \%$ for the brightest events in the sample ${ }^{5}$ ) was applied. The observer frame duration $T_{90}$, the time interval over which a burst emits from $5 \%$ to $95 \%$ of its total measured counts, was obtained and integrated burst counts over the whole event as well as the peak counts in the brightest $0.25 \mathrm{~s}$ interval were estimated. Due to the lack of spectral response, these detector counts cannot be directly converted into more physical units (fluences and peak fluxes).

For a quantitative evaluation of the uniformity of the SPIACS GRB sample we applied the $V / V_{\max }$ test (Schmidt 1968). In particular, $V / V_{\max }$ compares the volume in which the event was detected to the largest possible volume in which the event would be just at the selection threshold. The ratio of the volumes translates into the ratio of the peak counts, $C_{\mathrm{p}}$, over the minimum triggering counts, $C_{\min }$, in the same time interval as: $V / V_{\max }=\left(C_{\mathrm{p}} / C_{\min }\right)^{-3 / 2}$. The time intervals used range from the $0.05 \mathrm{~s}$ time resolution of SPI-ACS to the $T_{90}$ of the individual events.

In order to estimate the variability of the candidate burst light curves in the sample we used the variability formulation of Reichart et al. (2001), $V_{f=0.45}$. This measure is computed from the variance of the $50 \mathrm{~ms}$ binned light curve with respect to a boxcar-smoothed version of itself. Following previous attempts, we used $t_{45}$ as the smoothing time scale; this is the effective time for which the integrated counts of the brightest parts of the event are $45 \%$ of the total counts. The deviation of

degradation of the crystal structure by the space environment (cosmic rays, solar wind, etc.).

5 Note that the initial peak of the outburst of SGR1806-20 from Dec. 27, 2004, for which the SPI-ACS data were strongly affected by dead time effects, was about $10^{2}$ times stronger than the most luminous GRB in the sample. 

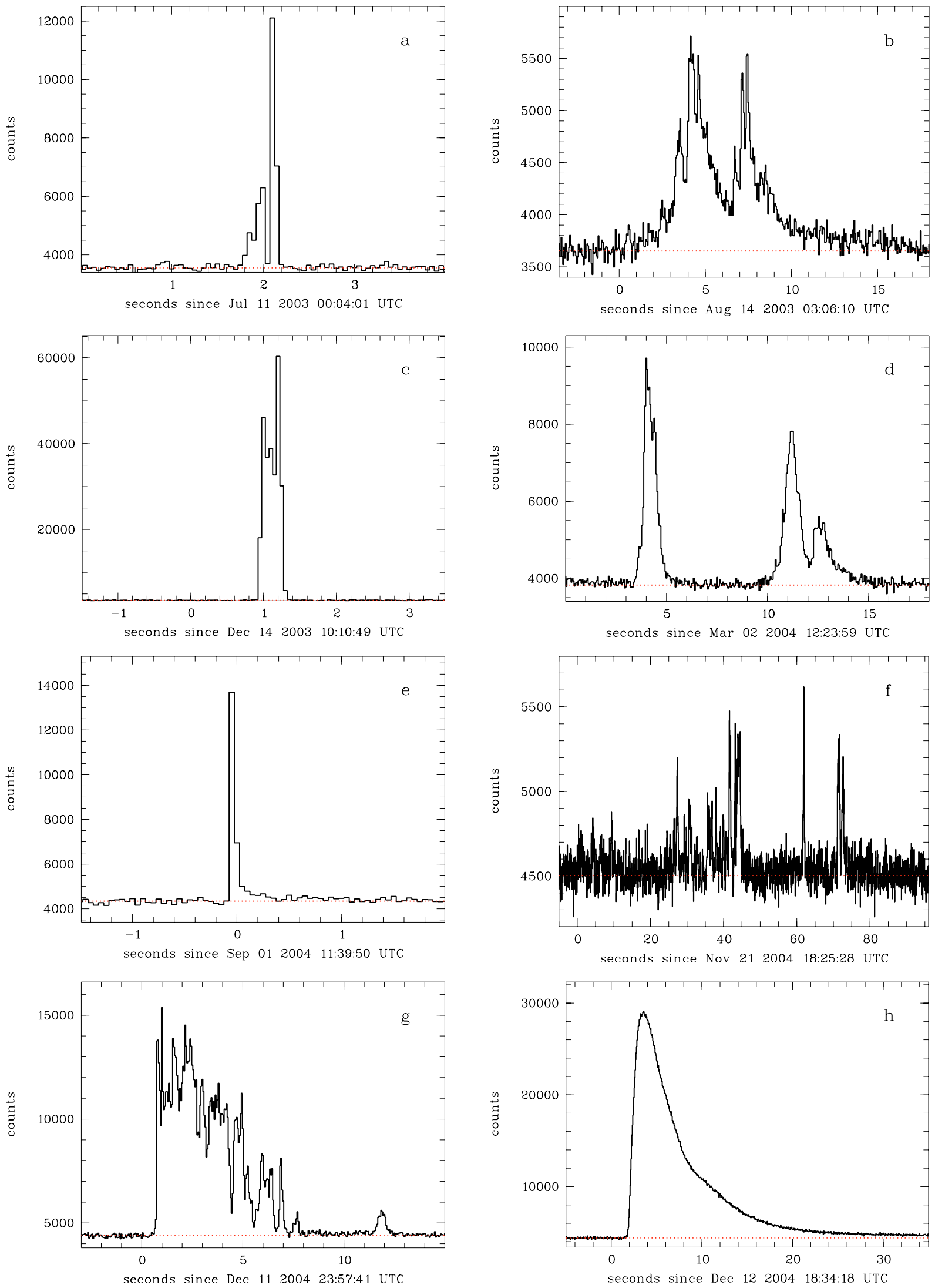

Fig. 2. SPI/ACS overall count rate light curves with $50 \mathrm{~ms}$ time binning for a selection of candidate GRBs from our sample. The horizontal dotted lines mark the background level. a) short GRB 030711. Note that triangulation cannot exclude SGR 0525-66 as the origin (Hurley et al. 2003a); b) long GRB 030814 (Hurley et al. 2003b); c) bright short GRB 031214 with a very hard $\left(E_{\mathrm{P}}=2000 \pm 80 \mathrm{keV}\right)$ spectrum (Hurley et al. 2003c; Golenetskii et al. 2003a,b); d) triple-peaked GRB 040302; e) bright unconfirmed event; f) multi-peaked GRB 041121; g) structured GRB 041211; h) very bright prototypical FRED (fast rise, exponential decay) GRB 041212. 
the SPI-ACS background from a Poissonian distribution was taken into account by broadening the distribution by a mean factor of 1.57 .

All 388 GRBs and candidates in the SPI-ACS sample are listed in Table 2 (available online at the CDS) together with the estimated burst properties. A continuously updated on-line version is available ${ }^{6}$ including figures and ASCII files of the light curves. The first column of Table 2 gives the number of the event in this catalogue. The trigger date (YYMMDD) and UTC are listed in the following two columns. The next four columns give the maximum significance of the event above the background, $T_{90}$, the total integrated counts in the event and the peak counts over $0.25 \mathrm{~s}$. The $V / V_{\max }$ statistic and variability measure are shown in columns eight and nine, respectively. The final column lists all other gamma-ray instruments and missions from which detections of the event were reported. Here, "U" stands for Ulysses, "K" for Konus/Wind, "M" for Mars Odyssey, "H" for HETE-2, "R" for RHESSI, "C" for Helicon/Coronas- $F$, "I" for INTEGRAL (SPI/IBIS), "S" for Swift and "X" for RXTE/ASM.

The table has numerous events with missing entries. For all GRBs which were confirmed by other instruments but detected by SPI-ACS below the sample selection threshold, only the time, date, significance and common instuments are listed. Furthermore, the variability measure was obtained only for long-duration events with sufficient signal-to-noise ratio.

\section{Results}

\subsection{Durations}

The distribution of the $T_{90}$ durations is shown in Fig. 3. For comparison the distribution derived from 1234 GRBs in the 4th BATSE GRB catalogue (Paciesas et al. 1999) was scaled to the elapsed mission time of SPI-ACS and included in the figure.

The distribution of the SPI-ACS burst candidates shows a bimodality similar to what was observed by the BATSE experiment. The long burst population has its maximum at $\sim 30 \mathrm{~s}$ and resembles the BATSE results closely. A short duration component is found in the SPI-ACS sample as well, but it deviates significantly from the BATSE sample. While the BATSE short burst $\log T_{90}$ distribution can be fit by a Gaussian centered at $T_{90}=0.45 \mathrm{~s}$ (Horvath 1998) the number of short burst candidates in the SPI-ACS sample rises steeply towards low values of $T_{90}$ and has its maximum at the time resolution of the instrument $(0.05 \mathrm{~s})$. Note that number of events with $T_{90} \leq 0.05 \mathrm{~s}$ has to be considered as a lower limit as events detected only in a single time bin are rejected already by the software which monitors the telemetry stream (see Sect. 2).

A further difference between the two samples is found in the ratios of short to long events. While the 4th BATSE catalogue included $\sim 70 \%$ long $\left(T_{90}>2 \mathrm{~s}\right)$ and $\sim 30 \%$ short bursts $\left(T_{90}<2 \mathrm{~s}\right)$, around $50 \%$ of the total events in the SPI-ACS sample have $T_{90}<2 \mathrm{~s}$. Note that the division into short and long bursts is not unambiguously defined, as different class boundaries are generally found when other properties (e.g.

\footnotetext{
${ }^{6}$ http://www .mpe.mpg.de/gamma/science/grb/ 1ACSburst.html
}

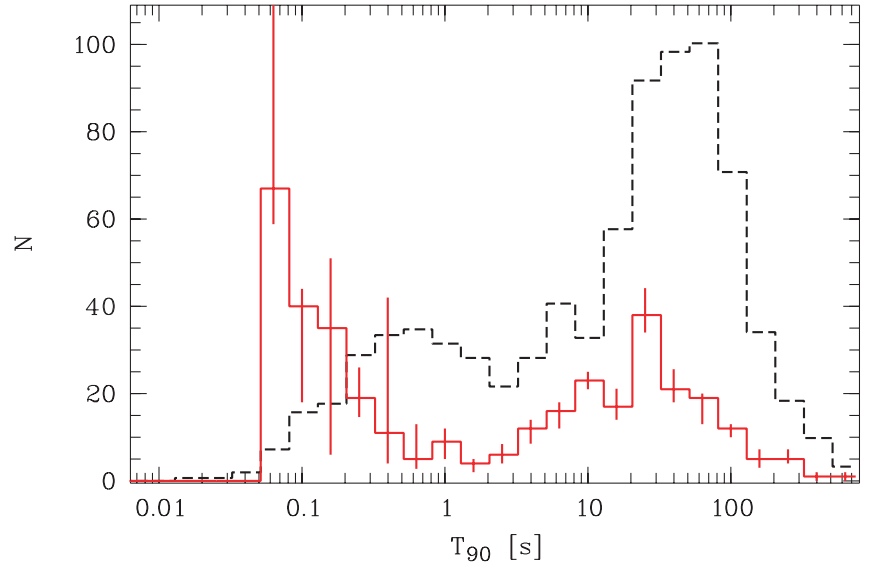

Fig. 3. Distribution of $T_{90}$ for all SPI-ACS GRB candidates (solid line) and for 1234 GRBs from the 4th BATSE GRB catalogue (Paciesas et al. 1999; dashed line) scaled to 21.6 months of continuous observation. Note the difference in the distributions of the short events.

fluence, spectral hardness ratios, $\left\langle V / V_{\max }\right\rangle$ are included (e.g. Mukherjee et al. 1998; Hakkila et al. 2000). In addition, the SPI-ACS and BATSE burst samples have been compiled using inherently different trigger and instrumental characteristics which imply different definitions for the short and long classes in both instruments.

The total number of detected events in SPI-ACS is of the order of $50 \%$ of the BATSE detections, when scaled to a common time interval. On the other hand, BATSE and SPI-ACS are sensitive to different energy bands. The SPI-ACS lower energy threshold of $\sim 80 \mathrm{keV}$ reduces the detection capability for bursts with peak energies below that energy. This affects mainly X-ray flashes $\left(E_{\mathrm{p}}<30 \mathrm{keV}\right)$ and X-ray rich bursts $\left(E_{\mathrm{p}} \sim 70 \mathrm{keV}\right)$. For comparison, BATSE could detect events down to $E_{\mathrm{p}}<25 \mathrm{keV}$ and was therefore capable of detecting a significant fraction of the X-ray rich burst population.

Figure 3 indicates a slight shift of the long-duration distribution components in SPI-ACS towards lower values of $T_{90}$ compared to the BATSE sample. Again, this could be a result of the lower sensitivity at softer energies for the long (and generally spectrally soft) distribution. In addition, it is typical to find a hard-to-soft evolution during the prompt emission phase. This also leads to shorter durations at higher energies. Finally, measurements of burst properties are instrument dependent, primarily due to siginal-to-noise limitations and temporal binning. This is likely an additional reason for the difference in the observed duration distributions.

The SPI-ACS short-duration population can be fit by a Gaussian with a maximum at the time resolution of $50 \mathrm{~ms}$. This shift with respect to the BATSE result is not of instrumental origin, as the SPI-ACS detectors are very sensitive at the typical peak energies of short GRBs. Also, a significant hard-to-soft evolution is not expected to occur and cause this offset. The deviations seem to be mainly for events with $T_{90}<0.25 \mathrm{~s}$. The general lack of spatial information in the data makes it difficult to determine the origin of these events. In Fig. 4 we show the duration distribution for the sample of confirmed GRBs. While $\sim 70 \%$ of the long-duration bursts are confirmed, less than $11 \%$ 


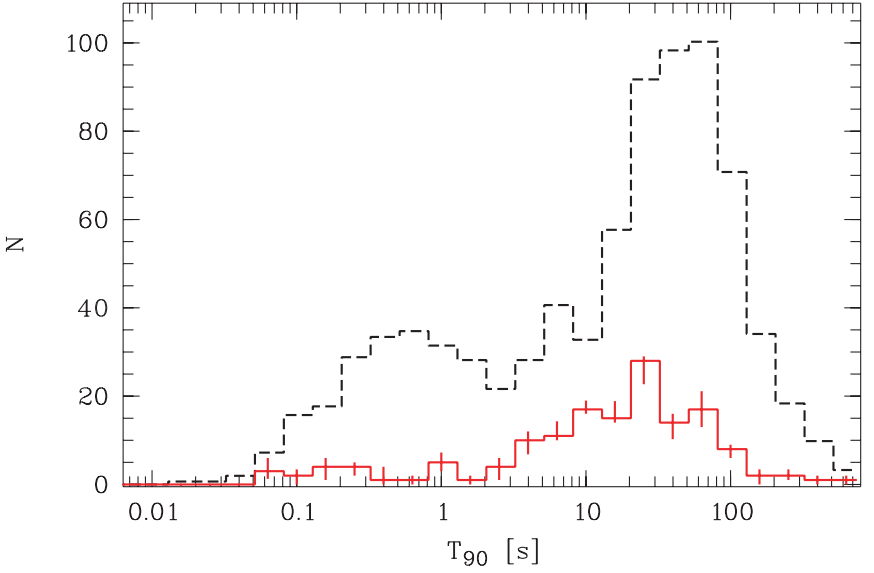

Fig. 4. Same as Fig. 3 except that only the confirmed bursts from the SPI-ACS sample are shown. A comparison with Fig. 3 reveals that for most of the long-duration bursts a cosmic origin was confirmed while this is true only for a small fraction of the short events.

( $8 \%)$ of the GRB candidates with $T_{90}<2$ s $(<0.25 \mathrm{~s})$ were detected by other gamma-ray instruments.

Note that we found no evidence of the proposed third (intermediate duration) burst population whose signature was detected in the BATSE sample (Horvath 1998). This population was claimed to to have a softer spectrum than the long-duration GRB sample. Due to the variety of trigger timescales used by SPI-ACS and the lack of spectral information, it is not clear how the temporal sensitivity depends on the spectral hardness and peak flux in the trigger. Thus, it apears difficult to ascertain whether SPI-ACS is indeed insensitive to the intermediate GRBs or whether some intermediate GRBs are included in the sample but for some reason not clearly distinguished from other bursts.

\subsection{The origin of events with $T_{90}<0.25 \mathrm{~s}$}

There are various possible explanations for the significant excess of unconfirmed $0.05-0.25 \mathrm{~s}$ long events in the SPI-ACS sample (an example is shown in Fig. 2e). We list them below, and present arguments for and against each of them.

(i) SPI-ACS is observing a real population of short, spectrally very hard GRBs, most of which must have been undetected by BATSE. However, the trigger efficiency of BATSE was quite sensitive to hard photons (Pendleton et al. 1998) and these short hard events would certainly have been seen. Another argument against the idea of observing a real burst population is that several gammaray instruments in orbit have the capability to detect such hard events, as demonstrated by the Mars Odyssey, Konus/Wind and Helicon/Coronas- $F$ detection of an event from December 14, 2003 (Fig. 2c); the number of such confirmations, however, is too low to explain the excess of very short events in this way.

(ii) A small contribution to the short burst population might come from outbursts of Soft Gamma Repeaters. These objects are strongly magnetized neutron stars, ("magnetars" - Duncan \& Thompson 1992), which are characterized by

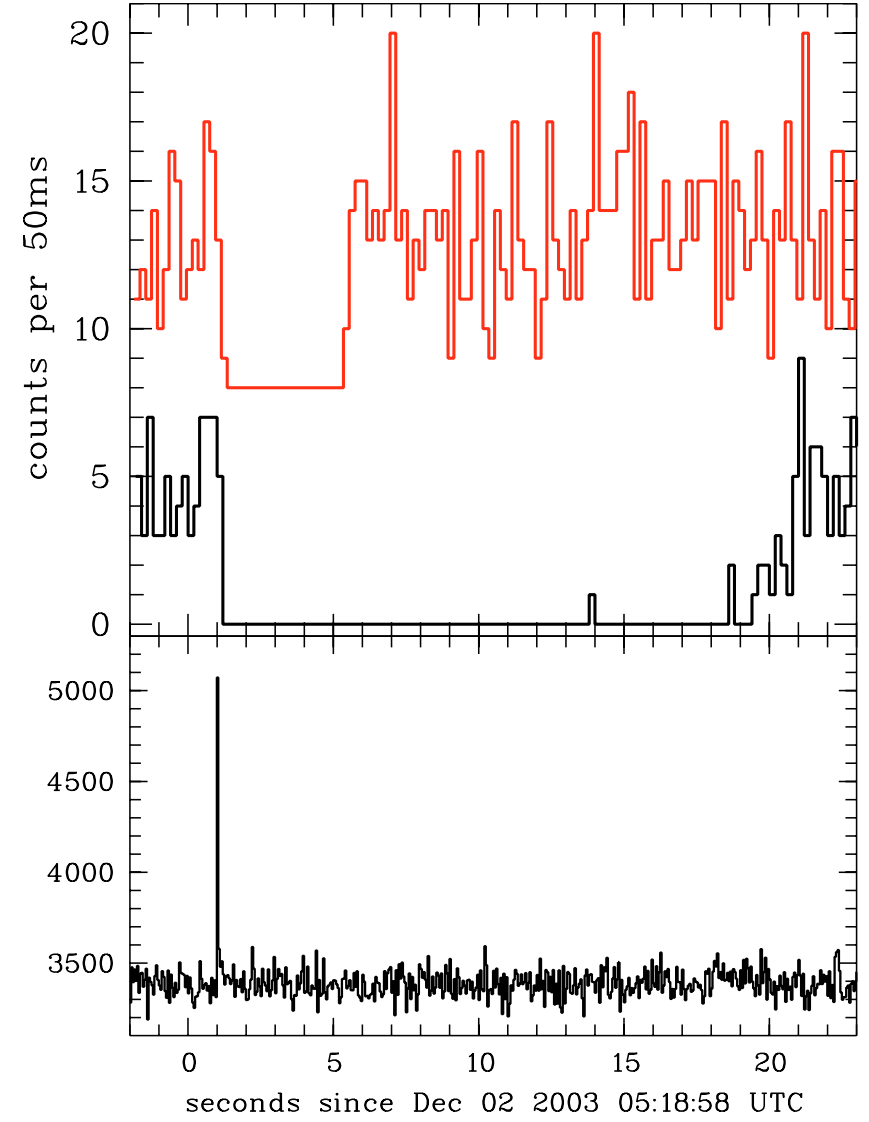

Fig. 5. Example of a very short event in SPI-ACS (bottom panel) with simultaneous saturation of two SPI Ge detectors (top panel). The SPIACS light curve is shown with $50 \mathrm{~ms}$ binning and the SPI light curves of detector \#9 (upper curve) and \#10 (lower curve) are binned with $200 \mathrm{~ms}$ time resolution.

brief (typical $\sim 100 \mathrm{~ms}$ ), very intense (up to $10^{44-45} \mathrm{erg}$ ) bursts of hard X-rays and soft gamma-rays. Their light curves resemble those of the short GRBs. Thus, events in the SPI-ACS count rate originating from known SGRs are not easily distinguishable from short GRBs without the localization by triangulation with other instruments. However, the vast majority of these bursts have soft spectra and most of them are not detected by the SPI-ACS.

(iii) The evaporation of primordial black holes through Hawking radiation can lead to a sudden burst of gammarays with durations $\sim 100 \mathrm{~ms}$ (Halzen et al. 1991). This has been proposed for the BATSE sample of very short bursts by Cline et al. (2003). Our sample of events with $T_{90}<100 \mathrm{~ms}$ is consistent with isotropy in an Euclidean geometry, $\left\langle V / V_{\max }\right\rangle \sim 0.48$. Recent re-analysis of the BATSE data found a value in agreement with SPI-ACS of $\left\langle V / V_{\max }\right\rangle=0.52 \pm 0.05$ (D. Cline, private communication).

(iv) A significant contribution to the short candidates could arise from instrumental effects and/or cosmic ray events. Analyzing the data of very short events together with simultaneous data from the spectrometer SPI revealed that a significant fraction of them are accompanied by the simultaneous saturation of one or several Ge-detectors. 
An example for an event on Dec. 1, 2003 is shown in Fig. 5. At the time of the significant rate increase in SPIACS over $\sim 50 \mathrm{~ms}$ two neighboring Ge-detectors, \#9 and \#10, went into a saturation which lasted for $\sim 4$ and $\sim 20 \mathrm{~s}$, respectively. The analysis of SPI data from a subset of satellite revolutions shows that a saturation event occurs approximately every four hours and that nearly all of these events have a simultaneous rate increase in SPI-ACS. The opposite approach, an independent search for short events in the SPI-ACS overall count rate, revealed $\sim 15$ events per hour at a $4.5 \sigma$ level. Combining these results shows that approximately every 60th very short SPI-ACS event coincides with a saturation in the Ge detectors.

The saturation of the Ge detectors can be explained by the deposit of a large amount of energy in the crystals. For example, a very energetic cosmic ray particle can traverse a BGO crystal and deposit part of its energy, thus producing the short count rate increase. Depending on the flight direction, the particle can go on to traverse one or several Ge detectors and cause the saturation, or pass through the BGO shield a second time, or not interact with any of the detectors again. The probability of a particle hitting the SPI-ACS and Ge detectors can be estimated from the geometry of the instrument to be $\sim 1 / 40$ of the probability to pass only through the anti-coincidence shield. This is in rough agreement with the rate of SPI-ACS short events that coincide with saturations. Therefore, this simple hypothesis suggests that a significant fraction of the unconfirmed short burst candidates can originate from cosmic ray particles hitting the instrument.

Most of the saturation events occur in a single Ge-detector. Hits in several detectors are significantly less frequent (Fig. 6) but happen either in neighboring detectors or along a line of detectors. If the saturation is caused by a single particle then a particle passing through the coded mask will only deposit energy in one detector as the cross-section for hitting more than one Ge-detector is low. A particle hitting the detector plane from the side can deposit energy (and cause saturation) in several detectors. This is in agreement with the observation that saturation events in more than one detector are always accompanied by a significant rate increase in the SPI-ACS, while a small fraction of the saturation events in single detectors show no corresponding signal in the SPI-ACS overall rate.

In the case of a particle shower, the number of detectors being hit depends on the width of the distribution of the secondary particles and on the inclination angle. In IBIS/PICsIT strong bursts ( 2500 counts) up to $170 \mathrm{~ms}$ long of particle-induced showers are observed (Segreto 2002). These showers may be produced by cosmic ray hits on the satellite or detector structure. As a consequence of the interaction of the primary particle with matter, high-energy photons and electron-positron pairs are created. These in turn produce a cascade of secondary electrons and photons via bremsstrahlung and pair production. The time profiles of the events observed in PICsIT are rather complex and both straight tracks and closed areas are visible (Segreto 2002). These observations are similar to the detections in SPI and thus support the idea of cosmic rays as a partial origin of the very short SPI-ACS events.

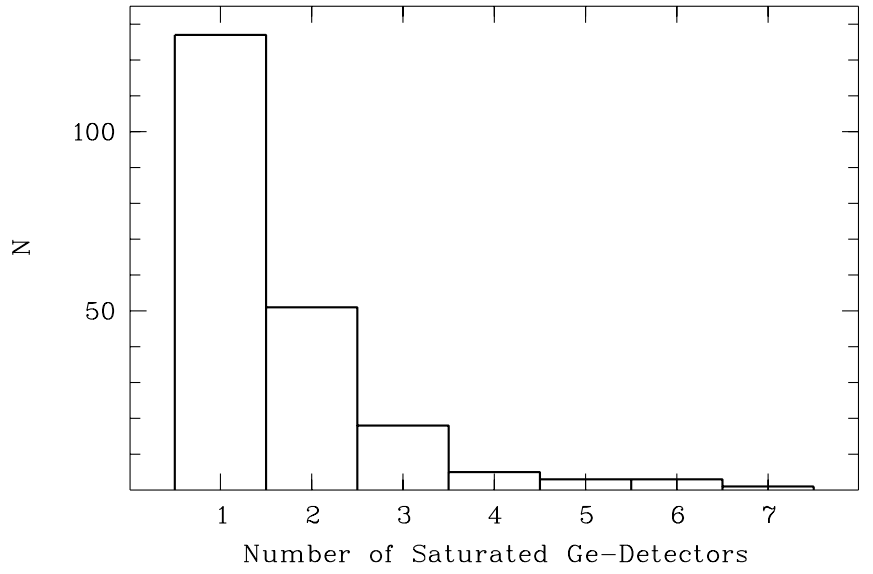

Fig. 6. Distribution of saturated Ge detectors of SPI for a duration of 15 days (INTEGRAL mission revolutions 137-141). Most saturations occur in a single detector only.

The observed rate increase in SPI-ACS can be produced by the deceleration of a cosmic ray particle in the BGO crystal. This induces a long-lasting $(50-150 \mathrm{~ms})$ phosphorescent afterglow energetic enough to cause recurrent triggers in the electronics. BGO was originally selected for SPI-ACS because of its short decay times and very low phosphorescence compared to e.g. $\mathrm{CsI}(\mathrm{Na})$ and $\mathrm{NaI}(\mathrm{Tl})$. BGO has decay times of $60 \mathrm{~ns}$ and $300 \mathrm{~ns}$ where the second is by far the more probable $(90 \%$ vs. $10 \%$ for the $60 \mathrm{~ns}$ ) and an afterglow of $0.005 \%$ is expected at $3 \mathrm{~ms}$ (Farukhi 1982).

In order to produce a single count in the SPI-ACS rate, a minimum of $80 \mathrm{keV}$ has to be accumulated during an integration time of $600 \mathrm{~ns}$. With a total light yield for BGO of 8000-10000 photons/MeV, this corresponds to $\sim 675$ photons. Assuming an exponential decay, the radiation will decrease to $0.005 \%$ at $3 \mu \mathrm{s}$. Thus the decay is so fast that only a small number of counts $(\sim 3)$ will be produced. From the afterglow properties the minimum original excitation energy can be estimated. For instance, to have 1000 recurrent triggers (a count rate increase of 1000) in a single crystal, the afterglow must be bright enough to be above the $80 \mathrm{keV}$ threshold for $\sim 3 \mathrm{~ms}$ (integration time + dead time). Thus, the $0.005 \%$ afterglow emission at $3 \mathrm{~ms}$ corresponds to a $\sim 1.6 \mathrm{GeV}$ initial excitation using the light yield given above. Therefore, particles which deposit $\sim 1.6 \mathrm{GeV}$ or more in a crystal can indeed be the origin of the short event population in the SPI-ACS. Short events showing similar temporal behavior have been discussed for $\mathrm{CsI}(\mathrm{Na})$ and CsI(T1) crystals exposed to primary cosmic radiation (Hurley 1978). The same conclusion was reached for the origin, namely cosmic ray nuclei in the iron group. Note that CsI has a significantly more intense afterglow $(0.1-1 \%$ at $6 \mathrm{~ms})$.

We performed simulations of particle attenuation in the detectors using $S R I M^{7}$. The results showed that heavy cosmic ray nuclei (e.g., Fe) with energies of several $\mathrm{GeV}$ per ion can easily produce the observed effects in the SPI-ACS and SPI.

\footnotetext{
${ }^{7}$ http://www.srim.org/
} 


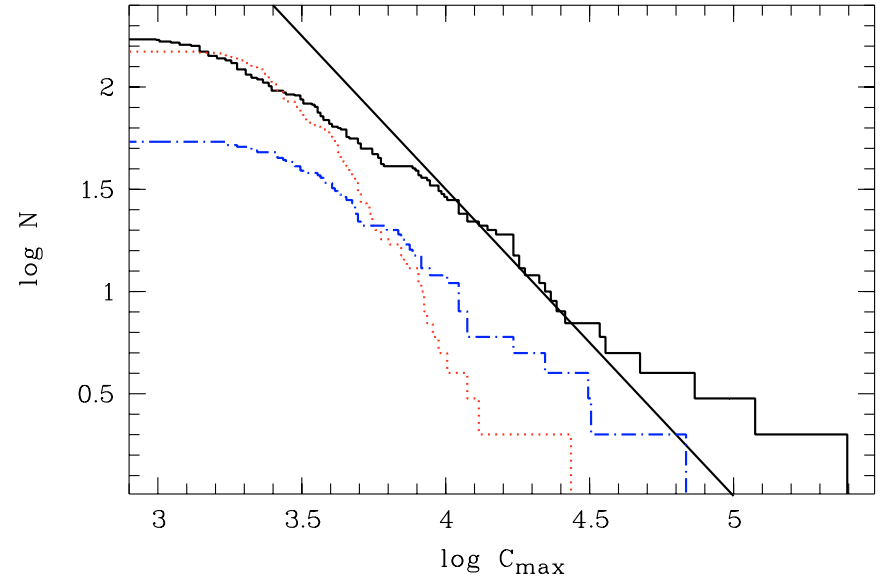

Fig. 7. $\log N-\log C_{\max }$ distribution for three sub-samples, together with the expected power law slope of $-3 / 2$ for a homogeneous distribution (solid line). The long duration $\left(T_{90}>2.5 \mathrm{~s}\right.$, solid line) and short duration $\left(0.25 \mathrm{~s}<T_{90}<2.5 \mathrm{~s}\right.$, dash-dotted line $)$ samples follow a similar power law with a slope of $\sim-1.1$. The very short events $\left(T_{90}<0.25 \mathrm{~s}\right.$, dotted) have a significantly steeper distribution.

\subsection{Intensity}

The distribution of burst intensities can provide valuable information on the radial distribution of the sources. The integral brightness distribution is expected to follow a power law with a slope of $-3 / 2$ for a homogeneous distribution of sources assuming a Euclidean geometry. The lack of spectral resolution limits the analysis for the SPI-ACS sample to detector count units. Figure 7 shows the integral $\log N-\log C_{\max }$ distributions for peak counts $C_{\max }$ of three sub-samples measured over a timescale of $0.25 \mathrm{~s}$. The solid, dashed and dotted distributions represent the long duration sample $(>2.5 \mathrm{~s})$, the short bursts $\left(0.25 \mathrm{~s} \leq T_{90}<2.5 \mathrm{~s}\right)$ and the population of very short events $(<0.25 \mathrm{~s})$, respectively. The deviation from a $-3 / 2$ power law (solid line) is visible for each of the subsamples. Both the long duration and the short duration bursts follow a $\sim-1.1$ slope. The population of very short burst behaves very differently, with a significantly steeper distribution (slope $\sim-2.3$ ). The turnover at low values of $C_{\max }$ corresponds to the selection threshold of the sample.

A deviation from a homogeneous distribution was also found by BATSE (Fishman et al. 1994). Nevertheless, the substitution of peak counts for peak fluxes causes an unknown uncertainty in the SPI-ACS results. While in a first-order approach each incoming photon produces one detector count, the uncertain effective area (depending on the incident angle) prevents a direct comparison. Thus, $C_{\max }$ depends on the spectral shape of the individual events. Still, a qualitative statement on the behavior of the three sub-samples can be made. Both shortand long duration bursts follow the same slope while the distribution of the very short events differs strongly. This is consistent with the hypothesis that the very short events are not of cosmological origin.

Figure 8 shows the corresponding $\log N-\log C_{\text {int }}$ distribution. Here, the integrated detector counts were used as a measure of the fluences. A behavior similar to the sub-samples in the $\log N-\log C_{\max }$ distribution is found. The long- and short

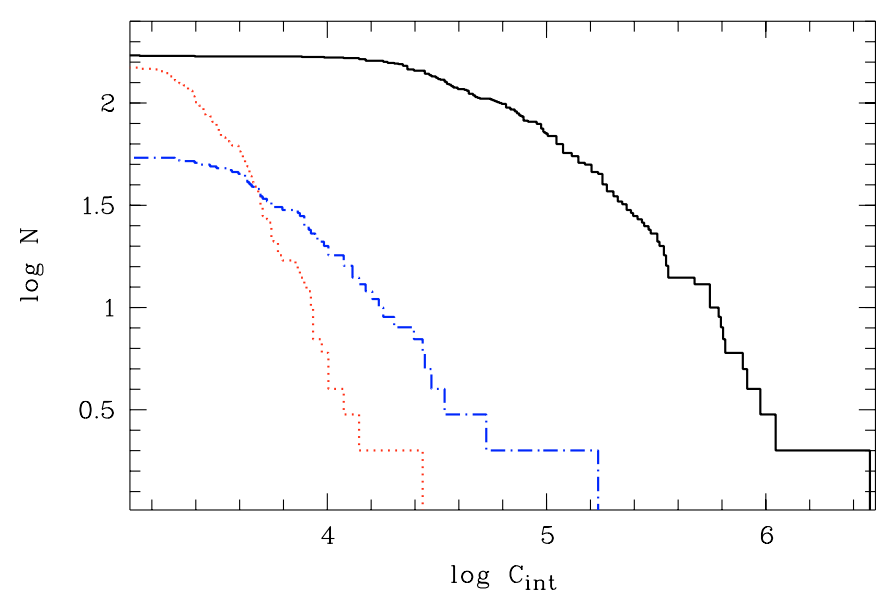

Fig. 8. Same as Fig. 7 for $\log N-\log C_{\text {int }}$.

duration populations evolve similarly while the sample of very short events displays a significantly steeper distribution.

\section{4. $V / V_{\max }$}

Another test for homogeneity is the $V / V_{\max }$ test (Schmidt 1968). If the burst population is drawn from a uniform distribution in Euclidean space, the mean value of $V / V_{\max }$ will be 0.5 . For the individual sub-samples of long, short and very short events we find $\left\langle V / V_{\max }\right\rangle$ values of $0.26,0.31$ and 0.48 , respectively. As already seen in the intensity distributions, the short and long burst populations behave similarly and show evidence for inhomogeneity. This is consistent with the BATSE result, namely $\left\langle V / V_{\max }\right\rangle \sim 0.34$ (Fishman et al. 1994). In contrast, $\left\langle V / V_{\max }\right\rangle$ for the very short events is consistent with a homogeneous distribution. Again, this points to a different origin for them compared to the short- and long duration bursts.

\subsection{Variability}

In Fig. 9 we show the distribution of the time profile variability of 143 candidate bursts using the measure $V_{f=0.45}$ presented by Reichart et al. (2001). The number of bursts decreases linearly with increasing variability following $N=(-74 \pm 3) \times V_{f=0.45}+$ $(33 \pm 0.8)$. The empirically found correlation between $V_{f=0.45}$ and the isotropic peak luminosity, $L_{\text {iso }}$ by Reichart et al. suggests that the majority of the events in the sample are intrinsically faint and only a few bursts have $L_{\text {iso }}>10^{53} \mathrm{erg}$.

\section{Conclusion}

The anti-coincidence shield of the INTEGRAL spectrometer SPI is operating successfully as an omnidirectional gamma-ray burst detector above $\sim 80 \mathrm{keV}$. The 1 st catalogue includes 388 candidate GRBs detected during the first 26.5 months of mission operation. For 179 triggers a cosmic origin could be confirmed by observations with other gamma-ray instruments in space. The sample shows the known duration bimodality with a strong excess at the very short end of the distribution $(<0.25 \mathrm{~s})$. The origin of this population of events ( $40 \%$ of the total number) was demonstrated to be significantly different from the 


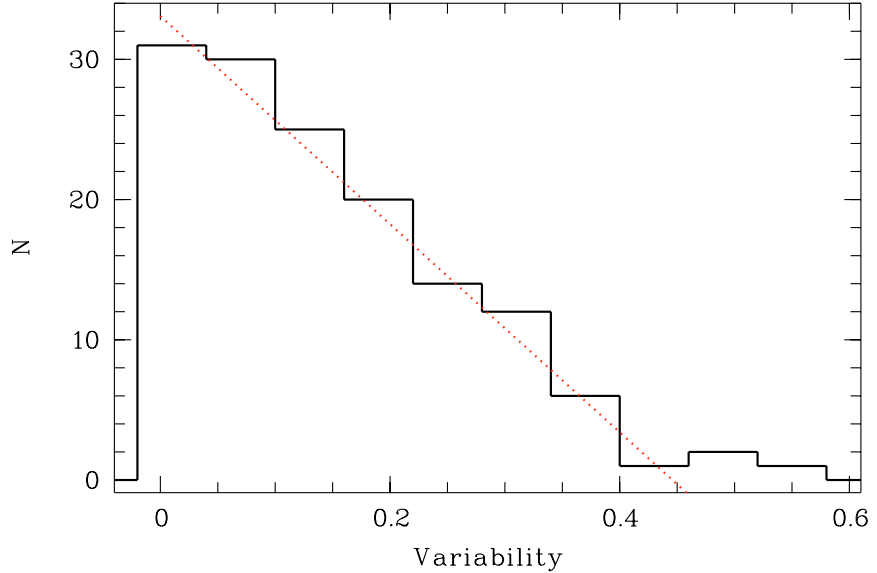

Fig. 9. Distribution of the variability measure for 143 candidate bursts from the sample (solid line) together with best linear fit (dotted).

normal GRB sample, as shown both by the $\log N-\log C_{\max }$ distribution as well as by the $V / V_{\max }$ test. Observations of simultaneous saturations in the spectrometer Ge detectors and very short events in the SPI-ACS overall rate suggest a cosmic ray origin for a significant fraction of these events.

The short- and long duration sample includes $236 \mathrm{GRBs}$, which corresponds to a detection rate of $\sim 130$ GRBs per year of continuous observation ( $\sim 1$ every three days). The intensity distribution of this sample is consistent with the BATSE results and shows a deviation from a homogenous distribution in Euclidean space.

Acknowledgements. We are grateful for the support from the ISDC shift team, especially to J. Borkowski and M. Beck. We thank the referee, J. Hakkila, for his insightful comments. The SPI-ACS is supported by the German "Ministerium für Bildung and Forschung" through the DLR grant 50.OG.9503.0. K.H. is grateful for support under the INTEGRAL US Guest Investigator program, NASA grants NAG5-12614 and NNG04GM50G. We are also grateful to V. Pal'shin for assistance in calibrating the ACS timing.

\section{References}

Band, D., Matteson, J., Ford, L., et al. 1993, ApJ, 413, 281

Cline, D. B., Matthey, C., \& Otwinowski, S. 2003, APh, 18, 531

Dezalay, J.-P., Lestrade, J., Barat, C., et al. 1996, ApJ, 471, L27

Duncan, R. C., \& Thompson, C. 1992, ApJ, 392, L9

Farukhi, M. R. 1982, IEEE, NS-29, 1237

Fishman, G. J., Meegan, C. A., Wilson, R. B., et al. 1989, in Proc. of the GRO Science Workshop, GSFC, 2

Fishman, G. J., Meegan, C. A., Wilson, R. B., et al. 1994, ApJS, 92, 229
Fishman, G. J., \& Meegan, C. A. 1995, ARA\&A, 33, 415

Golenetskii, S., Mazets, E., Pal'shin, V., \& Frederiks, D. 2003a, GCN, 2487

Golenetskii, S., Mazets, E., Pal'shin, V., \& Frederiks, D. 2003a, GCN, 2488

Hakkila, J., Haglin, D. J., Pendleton, G. N., et al. 2000, ApJ, 538, 165

Halzen, F., Zas, E, MacGibbon, J. H., \& Weekes, T. C. 1991, Nature, 353,807

Horvath, I. 1998, ApJ, 508, 757

Hurley, K. 1978, A\&A, 69, 313

Hurley, K. 1992, in Gamma-Ray Bursts - Proc. Gamma-Ray Burst Workshop - 1991, Huntsville, AL, ed. W. Paciesas, \& G. Fishman (New York: AIP Press), AIP Conf. Proc. 265, 3

Hurley, K. 1997, in Proc. of the 2nd INTEGRAL Workshop, ESA SP, 382,491

Hurley, K., Cline, T., Mitrofanov, I., et al. 2003a, GCN, 2296

Hurley, K., Cline, T., Mitrofanov, I., et al. 2003b, GCN, 2356

Hurley, K., Cline, T., Golenetskii, S., et al. 2003c, GCN, 2492

Kienlin, A. v., Arend, N., Lichti, G. G., Strong, A. W., \& Connell, P. 2003a, in Proc. of the SPIE, ed. J. E. Trümper, \& H. D. Tananbaum, 4851, 1336

Kienlin, A. v., Beckmann, V., Rau, A., et al. 2003b, A\&A, 411, L299

Klebesadel, R., Strong, I. B., \& Olsen, R. A. 1973, ApJ, 182, L85

Kouveliotou, C., Meegan, C. A., Fishman, G. J., et al. 1993, ApJ, 413, L101

Lamb, D. Q., Donaghy, T. Q., \& Graziani, C. 2005, ApJ, 620, 355

Lichti, G. G., Georgii, R., von Kienlin, A., et al. 2000, in Proc. 5th Compton Symp., AIP Conf. Proc., 510, 722

Mazets, E., Golenetskii, S. V., Il'inskii, V. N., et al. 1981, Ap\&SS, 80, 119

Mereghetti, S., Götz, D., Borkowski, J., et al. 2004, in Proc. 5th INTEGRAL Workshop, ESA SP, 552, 599

Mereghetti, S., Götz, D., von Kienlin, A., et al. 2005, ApJ, 624, L105

Mukherjee, S., Feigelson, E. D., Jogesh Babu, G., et al. 1998, ApJ, 508,314

Norris, J., Cline, T., Desai, U., \& Teegarden, B. 1984, Nature, 308, 434

Norris, J., Scargle, J., \& Bonnell, J. 2000, BAAS, 32(3), 1244

Paciesas, W. S., Meegan, C. A., Pendleton, G. N., et al. 1999, ApJS, 122,465

Pendleton, G. N., Hakkila, J., \& Meegan, C. A. 1998, in Proc. 4th Huntsville Symp., AIP Conf. Proc., 428, 899

Rau, A., von Kienlin, A., Lichti, G. G., Hurley, K., \& Beck, M. 2004, GCN, 2568

Reichart, D. E., Lamb, D. Q., Fenimore, E. E., et al. 2001, ApJ, 552, 57

Ryde, F., Borgonovo, L., Larsson, S., et al. 2003, A\&A, 411, L331

Schmidt, M. 1968, ApJ, 151, 393

Segreto, A. 2002, IN-IB-IASF/Pa-RP-030/02

Ubertini, P., Lebrun, F., Di Cocco, G., et al. 2003, A\&A, 411, L131

Vedrenne, G., Roques, J.-P., Schönfelder, V., et al. 2003, A\&A, 411, L63

Winkler, C., Courvoisier, T. J.-L., Di Cocco, G., et al. 2003, A\&A, 411, L1 